\title{
Scale-up of electrospray atomization using linear arrays of Taylor cones
}

\author{
Aaron J. Rulison ${ }^{\text {a) }}$ \\ Mechanical Engineering, California Institute of Technology, Pasadena, California 91125 \\ Richard C. Flagan \\ Chemical Enginecring 210-41, California Institute of Technology, Pasadena, California 91125
}

(Received 25 August 1992; accepted for publication 23 October 1992)

\begin{abstract}
Linear arrays of Taylor cones were established on capillary electrode tubes opposite a slotted flat plate counterelectrode to investigate the feasibility of increasing the liquid throughput rate in electrospray atomizers. It was found that individual Taylor cones could be established on each capillary over a wide range of the capillary radius to spacing ratio $R / S$. The onset potential $V_{s}$ required to establish the cones varied directly with $R / S$, but the liquid flow rate per cone and current per cone were nearly independent of $R / S$ for a given overpotential ratio $P=V / V_{s}$. Only six working capillaries were uscd, but the results per cone are applicable to larger arrays of cones since end effects were minimized.
\end{abstract}

\section{INTRODUCTION}

The electrospray disperses a liquid into droplets or ions by overcoming surface tension forces with electrical forces. Droplets or ions are emitted from the tip of a Taylor cone, ${ }^{1}$ which is the equilibrium shape of a free liquid surface at the end of a capillary electrode in a sufficiently intense electric field. It has been applied to production of ceramic powders, ${ }^{2,3}$ production of aerosol standards, ${ }^{4}$ application of coatings, ${ }^{5}$ dispersion of pesticides, ${ }^{6}$ space thrusters, ${ }^{7}$ and mass spectrometry. ${ }^{8}$ Technologies such as ceramic powder production by spray pyrolysis require large liquid throughputs to be economically feasible. A single Taylor cone, however, processes only several microliters per minute of typical ceramic precursors. ${ }^{2}$

The liquid throughput in electrospray atomization can be increased by operating a number of Taylor cones in parallel. This paper reports the liquid throughput and electrical current as a function of capillary spacing for a linear array of Taylor cones.

\section{EXPERIMENT}

A linear array of capillary electrodes opposite a slotted flat plate counterelectrode was constructed to test the operation of a multiple-cone sprayer (Fig. 1). Capillaries were made by squaring the tips of stainless steel hypodermic needles. Up to eight capillaries could be mounted with arbitrary spacing between two aluminum angle beams, one of which had a rubber strip on its face to provide holding friction. The counterelectrode was constructed from two stainless steel plates connected with a thin stainless steel sheet. A slot was maintained between the two plates by mounting them on a Lucite frame that also held the aluminum beams and capillaries. The capillaries were thus electrically insulated from the plates. A Spellman model

\footnotetext{
a) Now at the Jet Propulsion Laboratory 183-401, 4800 Oak Grove Drive, pasadena, CA 91109.
}

WRM15P1500D positive high voltage power supply was attached to the capillaries. The counterelectrode assembly was grounded through an ammeter sensitive to $\pm 10 \mathrm{nA}$.

Liquid was supplied to all capillaries using a single syringe pump emptying syringes in parallel. Thus, each capillary sprayed approximately the same volumetric flow rate of liquid. Although the capillaries were all at the same potential, they did not experience the same electric field due to end effects. To reduce the end cffccts, capillaries without liquid flow were added at each end of the array.

To establish the electrospray, the electrical potential on the capillaries was increased until Taylor cones formed on each capillary $V_{s}$ and then further increased to an overpotential ratio of either $P=V / V_{s}=1.39$ or 1.6. The liquid flow rate was adjusted to obtain a cone half angle of $37.5 \pm 2^{\circ}$ on the middle two cones in the array. The total electrical current $I_{\text {total }}$ was measured by the current arriving at the counterelectrode. The average current per cone was thus $I_{\text {total }} / n$, where $n$ is the number of capillaries supplied with liquid.

The liquid used in these tests was a solution of 0.618 $\mathrm{g} / \ell$ sodium iodide in $n$-propyl alcohol. The specific electrical conductivity was $0.00684 \mathrm{mho} / \mathrm{m}$. The surface tension was $0.0238 \mathrm{~N} / \mathrm{m}$.

\section{RESULTS AND DISCUSSION}

The electric field required to establish a Taylor cone at the end of a capillary (the electrode) opposite a flat plate (the counterelectrode) is ${ }^{9}$

$$
E_{s}\left(r_{c}\right)=\left(2 T \cos \theta / \epsilon_{0} r_{c}\right)^{1 / 2} \text {, }
$$

where $r_{c}$ is the capillary radius, $T$ is the liquid surface tension, $\theta$ is the cone semivertex angle, and $\epsilon_{0}$ is the permittivity of free space. The cone is only in equilibrium when the cone semivertex is $49.3^{\circ}$, ignoring space charge effects (De la Mora ${ }^{10}$ examines the role of space charge). 


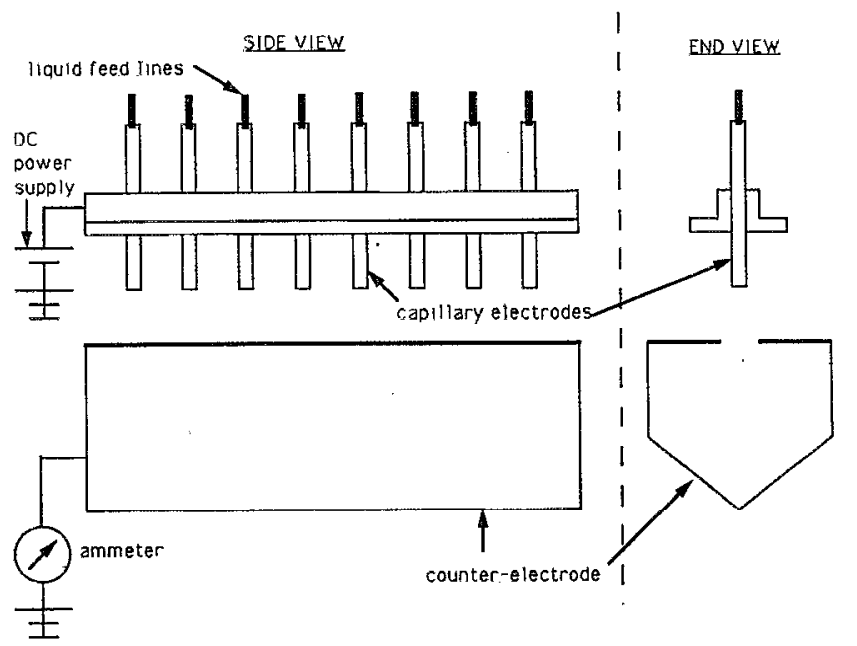

FIG. 1. Multiple electrode electrospray apparatus.

The potential difference between the electrode and counterelectrode required to reach this field strength is

$$
V_{s}=0.667\left(2 T r_{c} \cos \theta / \epsilon_{0}\right)^{1 / 2} \ln \left(4 h / r_{c}\right),
$$

where $h$ is the electrode to counterelectrode spacing. Thus, the required potential increases with increasing surface tension, electrode radius, and electrode to counterelectrode spacing.

With a lincar array of capillary electrodes the onset potential $V_{s}$ is greater than that for a single electrode since neighboring electrodes reduce the electric field strength. The dependence of the onset potential on $R / S$ is shown in Fig. 2. The $R / S=0$ case corresponds to infinite spacing and was approximated using a single capillary. The onset potential increased from 4800 to $7400 \mathrm{~V}$ as $R / S$ increased from 0 to 1.6. Thus closer capillary spacing required a higher potential to form Taylor cones.

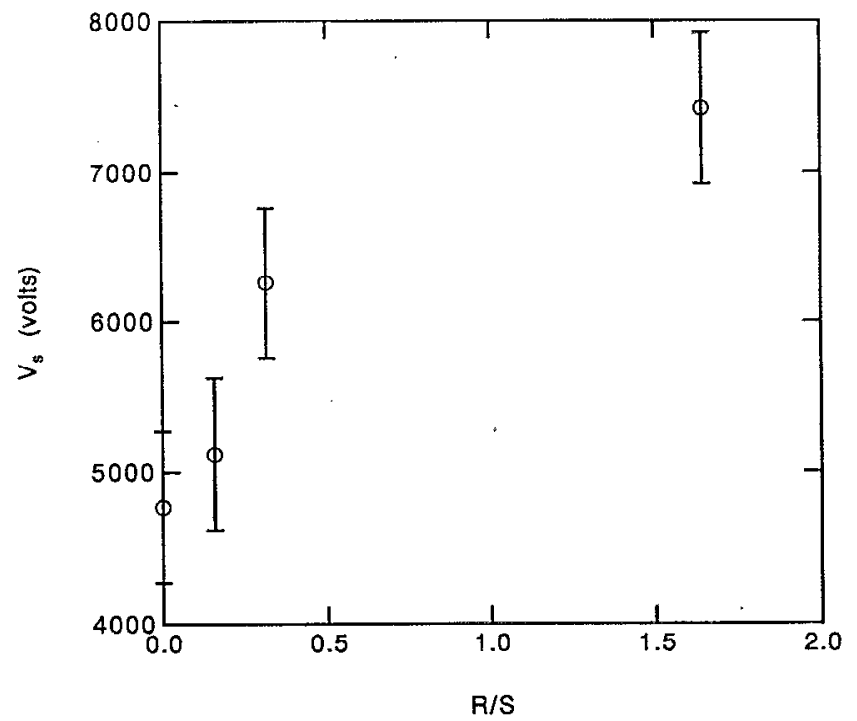

FIG. 2. Onset potential of Taylor cones vs capillary radius to spacing ratio.

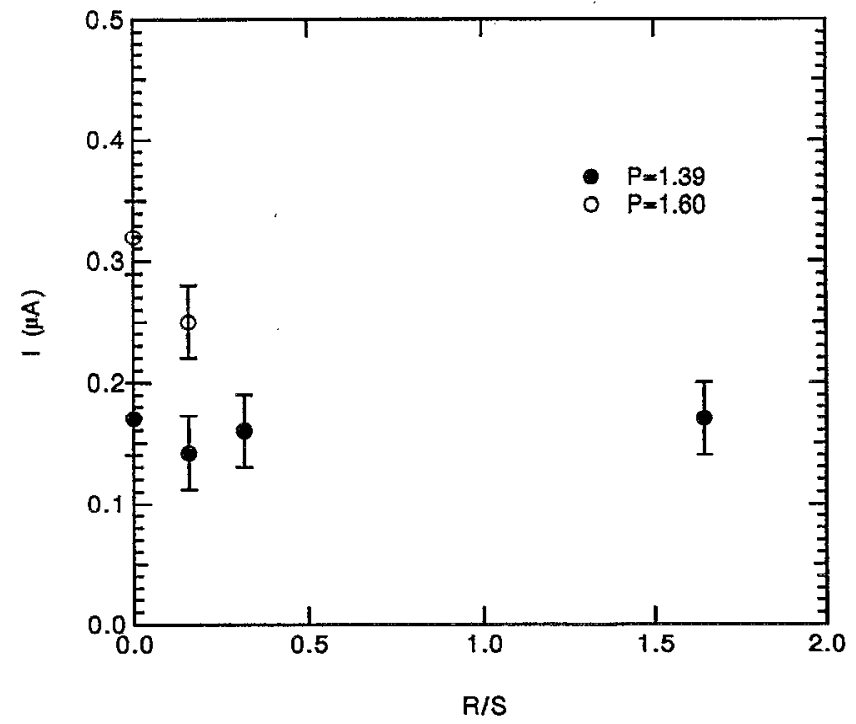

FIG. 3. Average electrical current per cone vs capillary radius to spacing ratio.

The liquid forming the cones responds to the electric field, not the potential level. Therefore, if the potential is set to give the same electric field at different values of $R / S$, the flow of liquid and current will remain the same, assuming that the asymmetric electric field components at each capillary are small compared to the symmetric ones. The measured value of $V_{s}$ provides an experimental determination of the electric field, and a reference value by which to set a constant electric field strength for operation of the cones. This was done by operating at fixed values of $P$ as $R / S$ varied.

The average current and volumetric flow rate per cone are plotted as functions of $R / S$ in Figs. 3 and 4 . For $P=1.39$, the current varied by only $25 \%$ as $R / S$ increased from 0 to 1.6. Similarly, the volumetric flow rate varied by

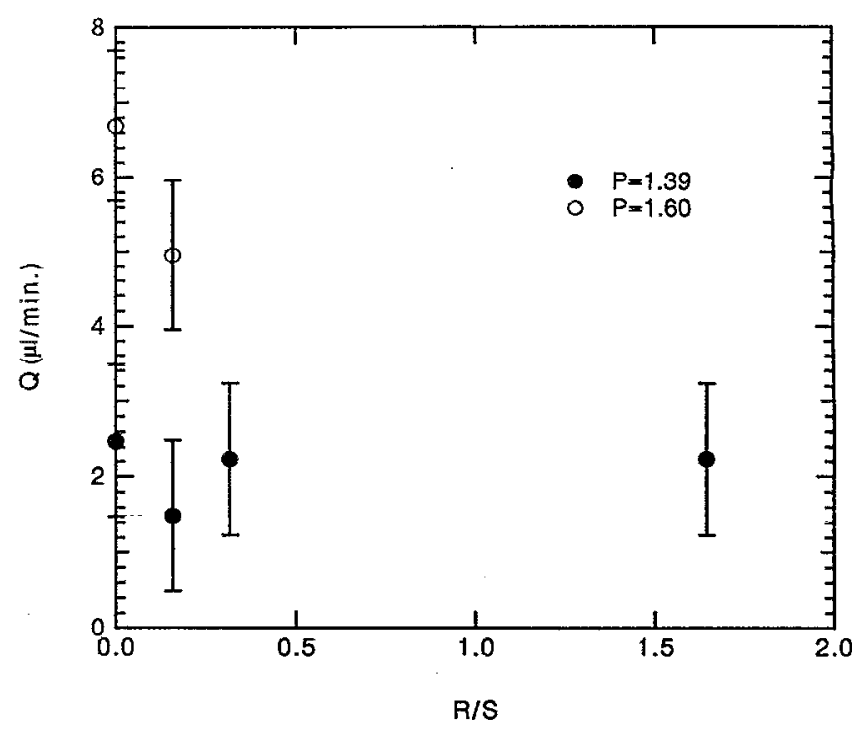

FIG. 4. Volumetric flow rate per cone vs capillary radius to spacing ratio. 

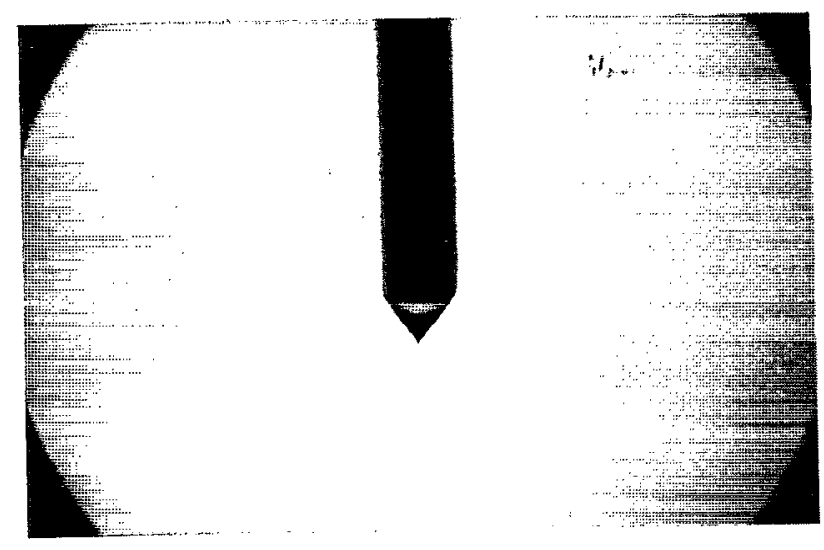

FIG. 5. Photograph of a Taylor cone established on a capillary electrode placed opposite a slotted flat plate electrode. $R / S \rightarrow 0, P=1.39$.

about $33 \%$. Most of the variation in current and volumetric flow rate was due to experimental inconsistencies in establishing the predetermined cone semivertex angle of $37.5 \pm 2^{\circ}$ by adjusting the volumetric flow rate. Note that the dip in current occurring at $R / S=0.2$ correlated with a dip in volumetric fiow rate.

Photographs of Taylor cones operating at $R / S \rightarrow 0$ and $R / S=0.31$ are shown in Figs. 5 through 7. Figure 5 shows a cone established on a single capillary, which corresponds to the case $R / S=0$. Neither the cone's shape nor its behavior differed noticeably from a cone established in an axially symmetric field, despite the asymmetric electric field applied to it by the slotted counterelectrode.

Figure 6 shows cones established on capillaries spaced such that $R / S=0.31$. Although the onset potential was greater for this array than for a single cone, and the proximity of the cones to one another introduced further asymmetries in the electric field, these cones did not differ noticeably in appearance or behavior from a cone established in an axially symmetric electrical field once they were established.

Figure 7 shows the right hand side of an array with cones established on capillaries spaced such that $R / S$
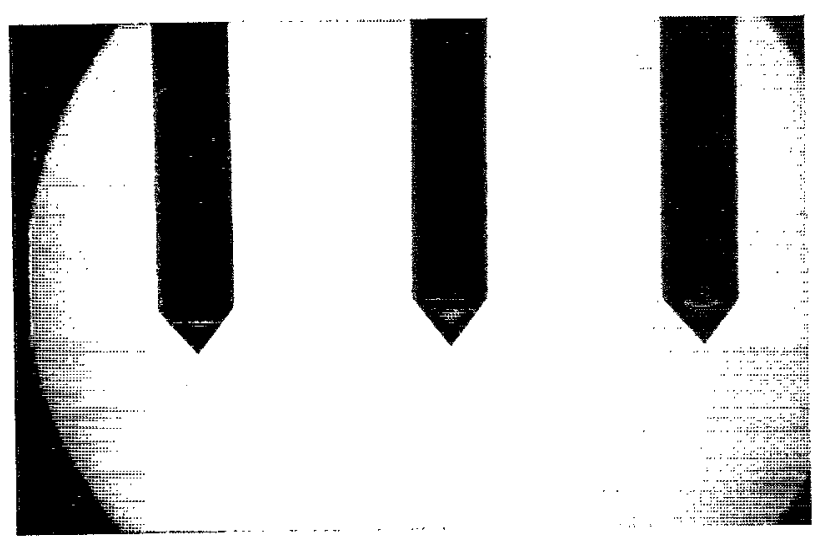

FIG. 6. Photograph of a linear array of Taylor cones established on capillary electrodes placed opposite a slotted flat plate electrode. $R / S$ $\Rightarrow 0.31, P=1.39$.
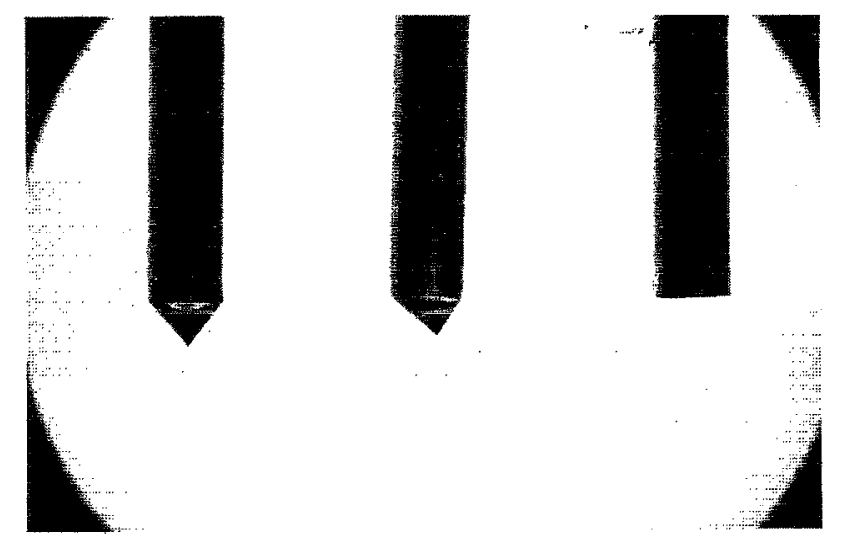

FIG. 7. Photograph of the right hand side of a linear array of Taylor cones established on capillary electrodes placed opposite a slotted flat plate electrode. $R / S=0.31, P=1.39$.

$=0.31$. The cones in the center of the array did not differ in appearance from a single cone. End effects, however, affect the cones located at the ends of the array. The end cone is deflected to the right, following the electric field. One end-effect capillary is present on each end of the array to reduce this effect. No liquid was supplied to the endeffect capillaries. To further reduce the field distortion, one might introduce conical tips on these guard electrodes. This would provide the influence of the Taylor cone, although it would not reproduce space charge effects.

\section{DISCUSSION}

Linear arrays of Taylor cones were established on capillary electrodes with various radius to spacing ratios $R / S$, placed opposite a slotted flat plate electrode. The onset potential needed to establish the cones varied inversely with capillary spacing. Operation of the cones at a constant overpotential ratio $P=V / V_{s}=1.39$ (independent of capillary spacing) resulted in nearly constant values of volumetric flow rate per cone and electrical current per cone, indicating that the electric field determines the electrospray properties and not the applied electrical potential, as expected.

Photographs show that the cones at the ends of the array were deflected due to end effects in the electric field. The feasibility, however, of establishing the electrospray at several capillary electrodes arranged in a linear array opposite a slotted flat plate is demonstrated. Larger numbers of capillaries could be accommodated using twodimensional arrays. This provides a means of greatly increasing the throughput of liquid in electrospray atomization.

\section{ACKNOWLEDGMENTS}

This work was supported by National Science Foundation (NSF) Grant No. CTS9113191.

${ }^{\prime}$ G. I. Taylor, Proc. R. Soc. London Ser. A 280, 383 (1964).

${ }^{2}$ A. J. Rulison and R. C. Flagan, J. Am. Ceram. Soc. (in press). 
${ }^{3}$ E. B. Slamovich and F. F, Lange, Mat. Res. Soc. Symp. Proc. 121, 257 (1988).

${ }^{4}$ G. M. H. Meesters, P. H. W. Vercoulen, J. C. M. Marijnissen, and B. Scarlett, J. Aerosol. Sci. 23, 37 (1992).

${ }^{5}$ A. E. Seaver and C. J. Eckhardt, U. S. Patent No. 4,748,043, 1988.

${ }^{6} \mathrm{~N}$. Morton, Crop protection 1, 27 (1982).
${ }^{7}$ C. Bartoli, H. von Rohden, S. P. Thompson, and J. Blommers, J. Phys. D 17, 2473 (1984).

${ }^{8}$ J. B. Fenn, M. Mann, C. K. Meng, S. K. Wong, and C. Whitehouse, Science 246, 64 (1989).

${ }^{9}$ D. P. H. Smith, IEEE Trans. Ind. Appl. IA 22, 527 (1986).

${ }^{10} \mathrm{~J}$. Fernandez de la Mora (private communication); J. Rosell-Llompart, J. Aerosol. Sci. 21, s673 (1990). 